\title{
Measuring the dihedral angle of water at a grain boundary in ice by an optical diffraction method
}

\author{
M. E. R. WALFORd AND J. F. NyE \\ H.H. Wills Physics Laboratory, University of Bristol, Bristol BS8 1TL, England
}

\begin{abstract}
Optical measurements have been made on the water lenses which form under pressure at grain boundaries in polycrystalline ice. Monochromatic light from a point source is focused by the lenses but, because the lenses are microscopic in size, the image is blurred by diffraction. The diffraction pattern observed under a microscope has been compared with the computed diffraction pattern to deduce the angle $2 \theta$ at the rim of each lens. This is the dihedral angle for water at a grain boundary in ice, and gives the ratio of the grain-boundary energy to that of an ice-water interface. The most sensitive measurements are those made on the rings of the virtual diffraction pattern formed on the object side of the lens. They give $\theta=12.5 \pm 0.5^{\circ}$ for the grain boundary under observation, which is $26 \%$ lower than the previous value for $\theta$ found by ignoring diffraction.
\end{abstract}

\section{INTRODUCTION}

When polycrystalline ice at the melting point is placed under stress, internal pressure melting occurs, with the formation of lens-shaped inclusions of water at some of the grain boundaries (Nye and Mae, 1972). Because of the ice-water surface energy, the lenses tend to have spherical faces; the lens diameters range from $400 \mu \mathrm{m}$ down to $20 \mu \mathrm{m}$ and below the level of optical measurement. The dihedral angle $2 \theta$ between the two spherical surfaces at the rim of each lens (Fig. 1) is determined by the surface energies of the grain boundary $\gamma_{\mathrm{gb}}$ and the ice-water interface $\gamma_{\text {iw }}$. Thus,

$$
2 \gamma_{\text {iw }} \cos \theta=\gamma_{\mathrm{gb}} .
$$

Optical measurements on such lenses by Walford (reported in Nye and Mae (1972)) and by Walford and others (1987) have been used to infer the angle $\theta$. This is of interest not only because it measures the ratio of the

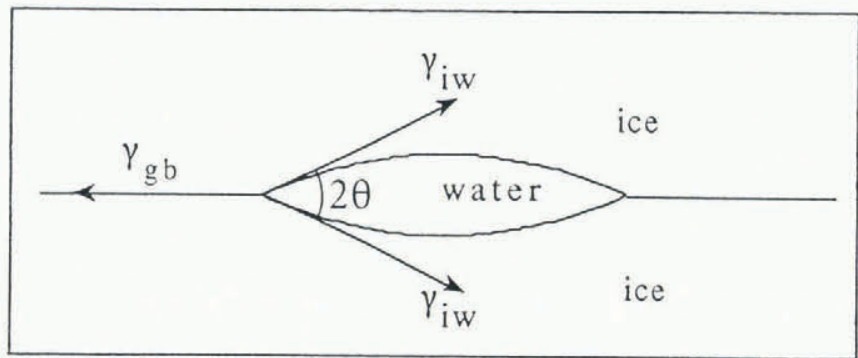

Fig. 1. A water lens at a grain boundary, showing the dihedral angle $2 \theta$. two surface energies, but also because it determines the cross-section of the veins of water which exist at the junctions where three grains meet (Nye and Frank, 1973), and therefore is relevant to the hydrology of temperate ice.

The method used was to pass parallel light through the lenses and measure their focal lengths. The refractive index of water is (slightly) greater than that of ice, and so the lenses are converging. Since the dihedral angle is about $30^{\circ}$, the lenses could be regarded as geometrically thick, but because the refractive-index ratio $\mu_{\text {iw }}$ is close to $1\left(\mu_{\mathrm{iw}}=1.0185\right)$ they are optically thin (this would be true up to dihedral angles of nearly $180^{\circ}$ ). Therefore, $\theta$ is expressed in terms of the focal length $f$, measured in ice, and the lens radius $a$ by the formula

$$
\sin \theta=\frac{a}{2 f\left(\mu_{\mathrm{iw}}-1\right)} .
$$

The experimental difficulty is to measure $f$ with sufficient accuracy in spite of the blurring of the focal spot by diffraction. Walford and his co-workers used a microscope to observe the position of the focus and, by measurements on 14 different lenses in two different fields, deduced a value for the dihedral angle of $2 \theta=33.6 \pm 0.7^{\circ}$.

It was apparent that a detailed calculation of the diffraction pattern produced by such a lens could allow a more accurate determination of the focal length. Viewed as an optical element, a water lens presents the unusual feature that light not only passes through it to produce the focus, but is also diffracted around it because the lens is not set in an opaque mount. The latter component of the light, if it were present by itself, would produce a 

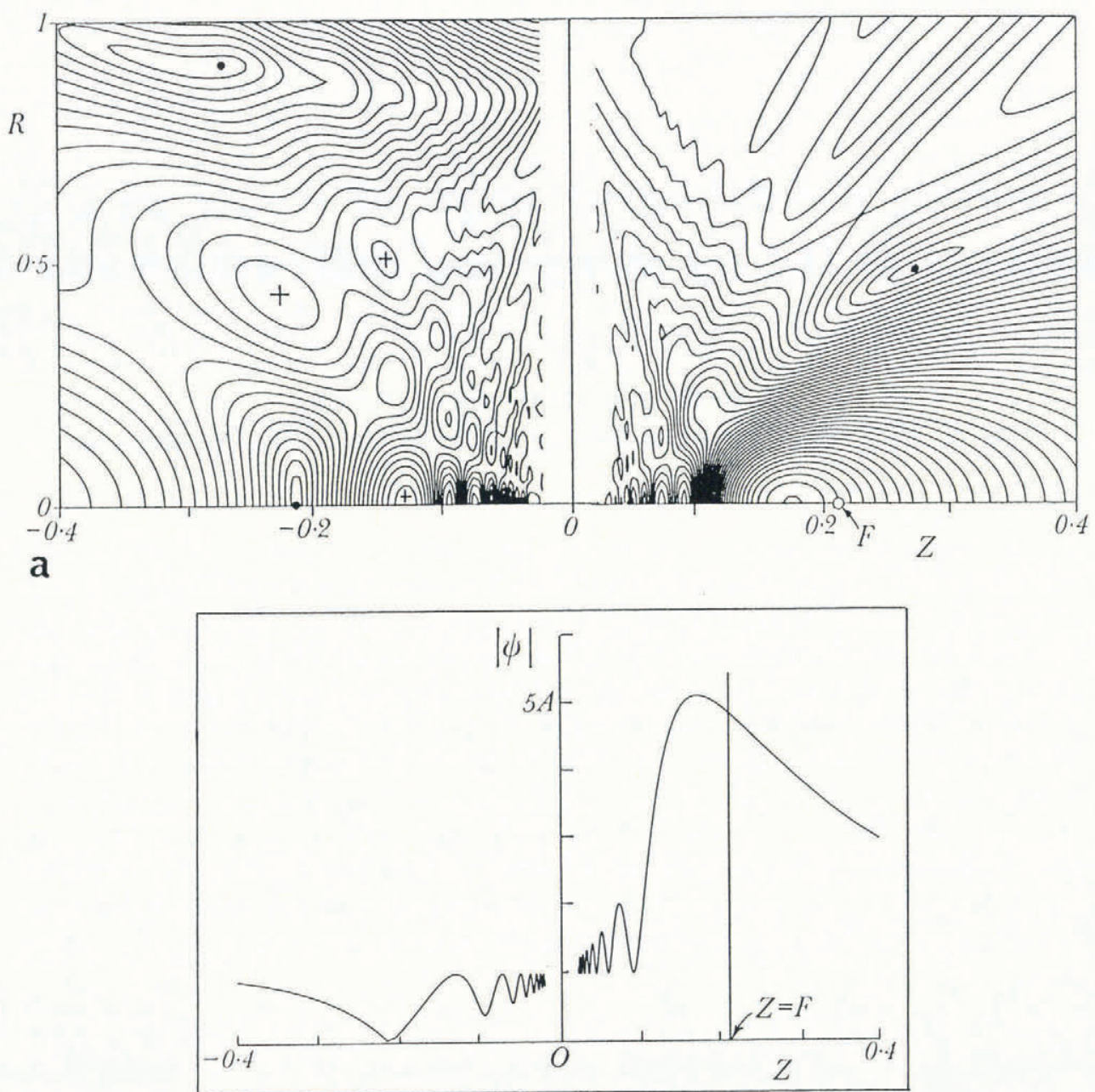

b

Fig. 2. The diffraction pattern formed by a small unstopped lens $(n=1.5)$ and a point source at infinity. (a) Contours of equal amplitude. The lens is at $Z=0$ with its edge at $R=1 ; Z=F$ is the geometrical focus. The black dots mark rings of zero amplitude. To help distinguish maxima from minima, some of the maxima in the virtual field (to the left of the lens) are marked by plus signs. With the amplitude at $Z=F$ taken as 1, the contour interval is 0.025 in the real field, and 0.01 in the virtual field, where the amplitudes are weaker. (b) The amplitude variation along the axis. Note the maximum of amplitude inside the focus, seen in both (a) and (b).

bright axial caustic, so that on a screen it would give the well-known Arago bright spot associated with diffraction by an opaque disc. When both components, light passing through the lens and around it, are present together they interfere, and the resulting three-dimensional diffraction pattern has been computed (Nye, 1991). A typical field is shown in Figure 2a and b and shows a number of interesting features. One of them is that the brightest point of the diffraction pattern is always closer to the lens than the geometrical image point (Farnell, 1957), and with lenses of the small size used in the measurements there is a serious systematic error of about $25 \%$ if the brightest point is taken as exactly indicating the geometrical image point. This effect is due to the small size of the lens rather than to the absence of a mount.

However, the position of the focal spot is not necessarily the feature of the diffraction pattern that is most sensitive to the dihedral angle. In principle, with the diffraction pattern known, any part of it may be used for a measurement of dihedral angle, and, moreover, the measurement does not have to be confined to the real part of the pattern. A microscope receiving the light from the lens and moved progressively nearer the lens will show a series of plane sections through the three-dimensional diffraction pattern. One of these will be through the geometrical focus, a closer one will be through the brightest point, moving closer again will show the plane of the lens itself, and closer still will appear sections through the virtual diffraction pattern. The pattern is virtual in the sense that it is not actually present behind the lens; however, a real image of a section of the virtual pattern is produced by the microscope, either on the retina of the observer's eye or on a photographic film. A detailed examination of the whole pattern showed that it is these sections through the virtual field that are most sensitive to variations in the dihedral angle.

\section{EXPERIMENTAL ARRANGEMENT}

Clear cylindrical ice specimens were prepared by slowly freezing distilled water, with constant stirring to prevent bubbles forming; the water was contained in a cylindri- 
cal polythene bag which was lowered at a rate of a few microns per second into a freezer at $-18^{\circ} \mathrm{C}$. The resulting ice had crystals a few millimetres across, considerably elongated in the growth direction. A sample was cut and transferred to a walk-in cold room held within $2 \mathrm{deg}$ of the melting point. There it was brought slowly to the melting point, compressed axially for a few hours at a stress of about 1 bar and left to anneal overnight in a polythene bag held in a dewar flask of ice and distilled water. This treatment produced a host of rather stable, separate, water lenses of up to a few hundred microns in diameter, appearing particularly at those grain boundaries which were normal to the compressive stress (Nye and Mae, 1972). We then reduced the sample by partially melting it against a warm metal plate to give a rectangular block approximately $2 \mathrm{~cm} \times 3 \mathrm{~cm} \times 5 \mathrm{~cm}$, with such a grain boundary parallel to its large face. The block was mounted within an optical-quality glass cell with the chosen grain boundary or lens plane horizontal, and held there by blocks of expanded polystyrene (Fig. 3). To avoid uneven refraction at the top and bottom surfaces, the block was covered with an ice/distilled water mixture, the refractive index contrast between ice and water being much smaller than that at the optically perfect interface between water and air.

The cell was placed on the stage of a cleaned and carefully aligned transmission optical microscope. The water lenses were examined in white light (which gives clear viewing) using a low-power objective (which has a conveniently long working distance). No sub-stage condenser was used, but a parallel-sided water cell was placed in the optical path to reduce unwanted radiation melting in the sample. A suitable candidate lens having been placed on the optical axis of the instrument using the $x-y$ stage controls, we checked the alignment by racking the microscope up and down; this was impor-

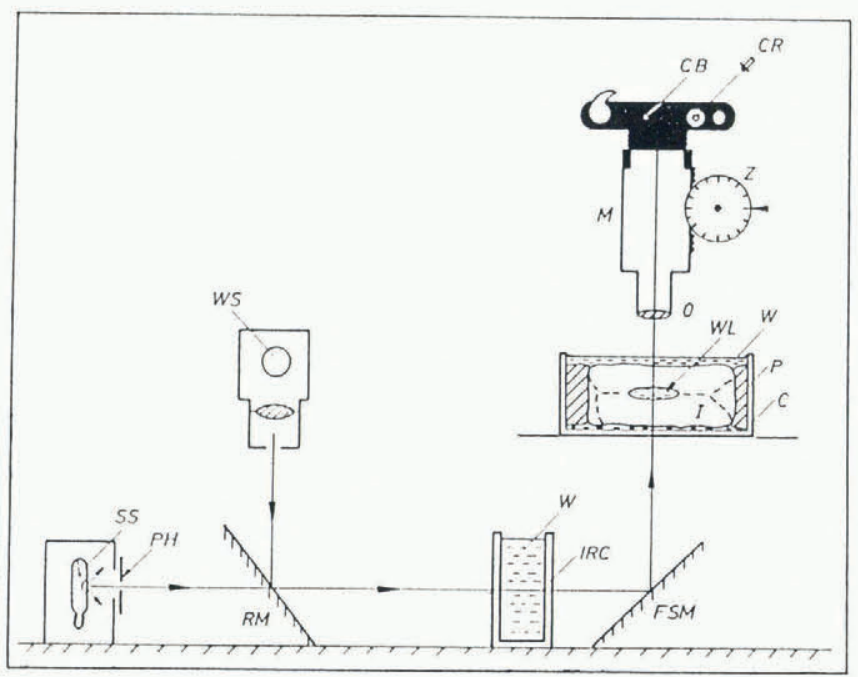

Fig. 3. The experimental apparatus. For clarity a single water lens only is shown (WL); its size relative to the rest of the apparatus is greatly exaggerated. C sample cell; $C B$ camera back; CR cable release; FSM front-silvered mirror; $O$ objective lens; $P$ polystyrene blocks; $\mathrm{PH}$ pinhole; $\mathrm{RM}$ removable mirror; SS sodium lamp; W water; WS white-light source; Z calibrated height control.
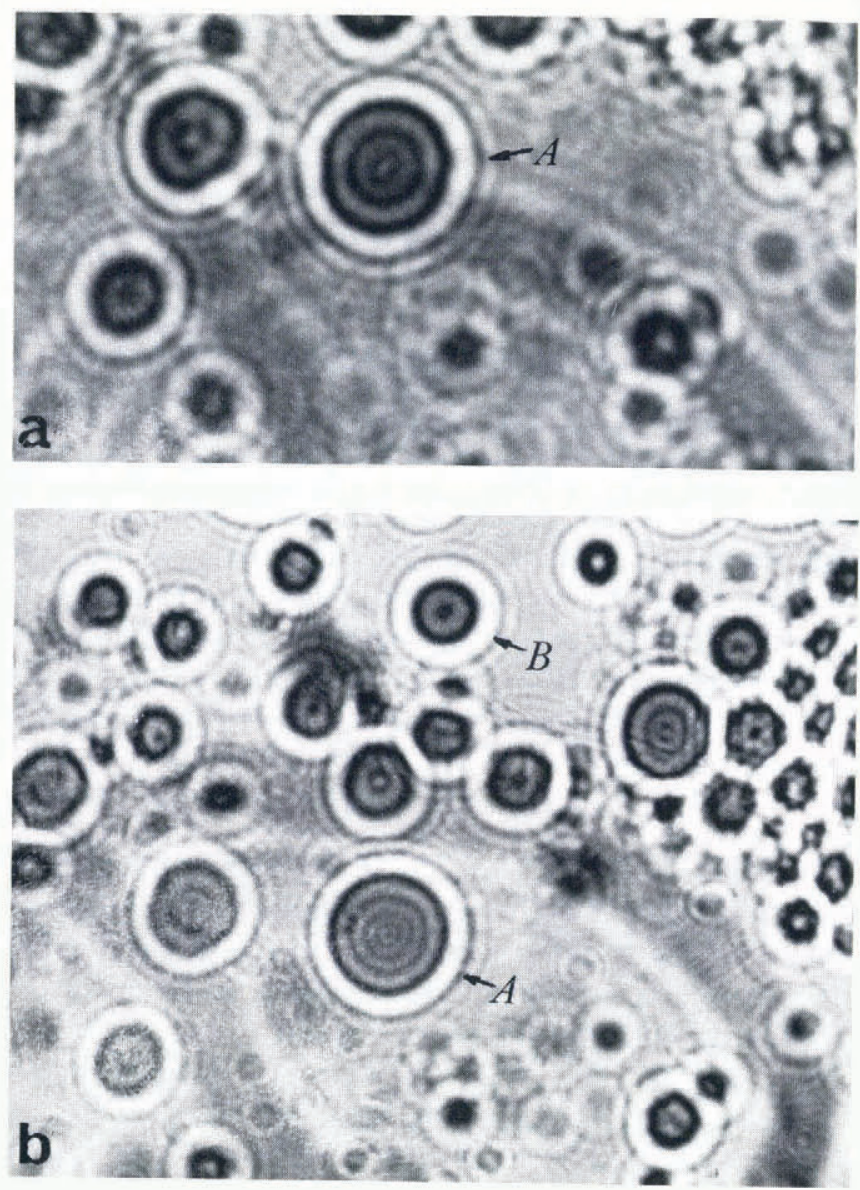

Fig. 4. Diffraction patterns produced by water lenses at a grain boundary in ice. Measurements were made of the virtual diffraction patterns produced by lenses $A$ and $B$ at distances (referred to paths in ice) $z=-22.58 \mathrm{~mm}$ (Fig. 4a) and $-12.99 \mathrm{~mm}$ (Fig. 4b). The lens radii are $a=182 \mu \mathrm{m}$ (lens $A$ ) and $96.3 \mu \mathrm{m}$ (lens B), and the calculated focal lengths in ice are $f=22.73 \mathrm{~mm}$ (lens $A$ ) and $12.03 \mathrm{~mm}$ (lens B).

tant to ensure that we should be able to photograph the diffraction pattern over a wide range of $z$ values without disturbing the specimen. We then switched to a highpower sodium lamp with a $2 \mathrm{~mm}$ stop, placed on the optical axis at a distance of $1.20 \mathrm{~m}$ and, after rechecking the alignment, replaced the microscope eyepiece with a camera back. The diffraction pattern was recorded on a fine-grain film. Some 40 photographs were taken at measured $z$ intervals of about $1 \mathrm{~mm}$ spanning both the real and the virtual fields. Because the entire operation took over an hour (each photograph needed an exposure time of $1 \mathrm{~min}$ ), we repeated the first photograph at frequent intervals to check that no significant changes had occurred. Usually the only significant effect was condensation on the bottom of the sample cell, because of the presence of the experimenter. This condensation was delicately removed with lens tissue as necessary. Finally, a $1 \mathrm{~mm}$ microscope graticule was photographed to record the magnification.

Figure $4 \mathrm{a}$ and $\mathrm{b}$ shows diffraction patterns at two different distances produced by the same lenses in one grain boundary, Figure $4 \mathrm{~b}$ including both the lenses selected 
for detailed analysis. Also visible are diffraction patterns produced by other lenses, by droplets of condensation and by water veins or surface grain-boundary grooves. It was because of these effects that we used sodium light in the experiments; with a laser, and its more coherent light, the diffraction rings of a single lens were highly confused by unwanted patterns.

\section{FITTING OBSERVATIONS TO THEORY}

\subsection{Summary of the theory}

The water lens is assumed to be set within an infinite medium (ice). The theory in Nye (1991) uses Kirchhoff diffraction theory in the paraxial approximation to calculate the complex wave amplitude $\psi$ due to a point source of monochromatic waves on the lens axis. $\psi$ is the sum of an inner part $\psi_{\mathrm{i}}$, due to light that has passed through the lens, and an outer part $\psi_{\mathrm{o}}$, due to light that has passed around the lens,

$$
\psi=\psi_{\mathrm{i}}+\psi_{\mathrm{o}}
$$

The wave front emerging from the lens consists of a spherical wave of radius $v$, converging on the geometrical image point and giving rise to $\psi_{\mathrm{i}}$; this is surrounded by an annular part of a spherical wave of radius $u$, diverging from the point source, which gives rise to $\psi_{0}$. Thus, $u$ and $v$ are (approximately) the axial distances of the object and image from the lens. The focal length $f$ is defined by the equation $f^{-1}=u^{-1}+v^{-1}$. All these distances are within the ice.

We take the origin $\mathrm{O}$ at the lens (for a more precise definition see the original paper) and cyclindrical polar coordinates $r, z$ with $z$ longitudinal along the axis in the direction of the light, and $r$ radial. Corresponding dimensionless quantities are defined as follows:

$$
\begin{array}{rlrl}
R=\frac{r}{a}, & Z=\frac{2}{k a^{2}} z, & F=\frac{2}{k a^{2}} f, \\
U & =\frac{2}{k a^{2}} u, \quad V=\frac{2}{k a^{2}} v
\end{array}
$$

where $a$ is the radius of the lens, $k=2 \pi / \lambda$, and $\lambda$ is the wavelength of the light in the ice. It is useful to express the dimensionless focal length $F$ in terms of the number $n$ of half-period Fresnel zones subtended by the lens at its focus,

$$
F=\frac{1}{n \pi}
$$

Then $\psi_{\mathrm{i}}$ and $\psi_{\mathrm{o}}$ are given by

$$
\begin{gathered}
\psi_{\mathrm{i}}(R, Z)=-\frac{2 i A}{Z} \exp i\left(\frac{k^{2} a^{2}}{2} Z+\frac{R^{2}}{Z}\right) \\
\cdot \int_{0}^{1} \mathrm{~d} \rho \rho \exp i\left\{n \pi\left(1-\rho^{2}\right)+\frac{\rho^{2}}{W}\right\} J_{0}\left(\frac{2 R}{Z} \rho\right) \\
\psi_{0}(R, Z)=-\frac{2 i A}{Z} \exp i\left(\frac{k^{2} a^{2}}{2} Z+\frac{R^{2}}{Z}\right) \\
\cdot\left[\frac{i W}{2} \exp \left(-\frac{i R^{2} W}{Z^{2}}\right)\right. \\
\left.-\int_{0}^{1} \mathrm{~d} \rho \rho \exp \left(\frac{i \rho^{2}}{W}\right) J_{0}\left(\frac{2 R}{Z} \rho\right)\right]
\end{gathered}
$$

Here $J_{0}$ is a Bessel function, $A$ (real) is the amplitude of the spherical wave incident on the lens, and $W$ is the dimensionless distance given by $W^{-1}=Z^{-1}+U^{-1}$. Thus, for an object at infinity, $W$ is identical with $Z$; in general, since $U$ is fixed, $W$ is a function of $Z$.

The exponential factor that appears before the integral sign in Equation (6) and before the square bracket in Equation (7) is a phase factor. For a given position $(R, Z)$ in the field, $\psi_{\mathrm{i}}$ depends only on $n$, apart from this phase factor, and $\psi_{\mathrm{o}}$ is independent of $n$. Thus, since the phase factor does not affect the intensity $|\psi|^{2}$, the field of intensity depends, apart from a geometrical scaling, only upon the single parameter $n$; for different $n$ the observed diffraction patterns will be essentially different.

\subsection{Relating the theory to the experiment}

To relate this theory to observation, we must first deal with the fact that in the theory the diffraction pattern is formed entirely within the ice, whereas the observations are made on water lenses contained within a parallelsided slab of ice, which is itself immersed in a water bath, with the microscope moving in air, and with the point source also in air. It is a matter of real and apparent depth. A detailed consideration of the additional refractions involved shows that the object and image sides have to be treated differently, essentially because the object distance was measured directly, whereas the depth of the observed cross-section through the diffraction pattern was obtained by noting the position of the microscope carriage along the axis. The object distance $u$ of the theory is related to the actual position of the source by

$$
u=\mu_{\mathrm{i}}\left(\frac{l_{\mathrm{o}}}{\mu_{\mathrm{o}}}+\frac{l_{\mathrm{w}}}{\mu_{\mathrm{w}}}+\frac{l_{\mathrm{i}}}{\mu_{\mathrm{i}}}\right)
$$

where $\mu_{\mathrm{o}}, \mu_{\mathrm{w}}$ and $\mu_{\mathrm{i}}$ are the refractive indices for air, water and ice, and $l_{\mathrm{o}}, l_{\mathrm{w}}$ and $l_{\mathrm{i}}$ are the lengths of the ray paths in these media ( $l_{\mathrm{w}}$ is very small and there is also a negligible path length through glass). On the other hand, the position $z$ in the diffraction field is related to the apparent distance $z^{\prime}$ as measured by the microscope scale by

$$
z=\frac{\mu_{\mathrm{i}}}{\mu_{\mathrm{o}}} z^{\prime},
$$

independently of the positions of the ice-water and water-air interfaces. This means, incidentally, that as the plane of focus of the microscope moves through the water-air interface, for example, there is no apparent sign of this in the diffraction pattern.

Note that, although the graticule used for finding the magnification was in air while the water lenses were in ice, no correction for the transverse magnification is needed.

\subsection{Treatment of the observations}

As described in section 2, observations were made on two lenses, $\mathrm{A}$ and $\mathrm{B}$, in the same grain boundary. The radius $a$ could have been measured from a single photograph taken in the plane of the lens, but the difficulty with this method is that the lens almost disappears from view precisely when the microscope is focused on its plane. It was preferable to deduce $a$ by interpolation of observations made on either side of the plane of the lens. This gave $a=182 \mu \mathrm{m}$ for lens A and $a=96.3 \mu \mathrm{m}$ for lens B. 


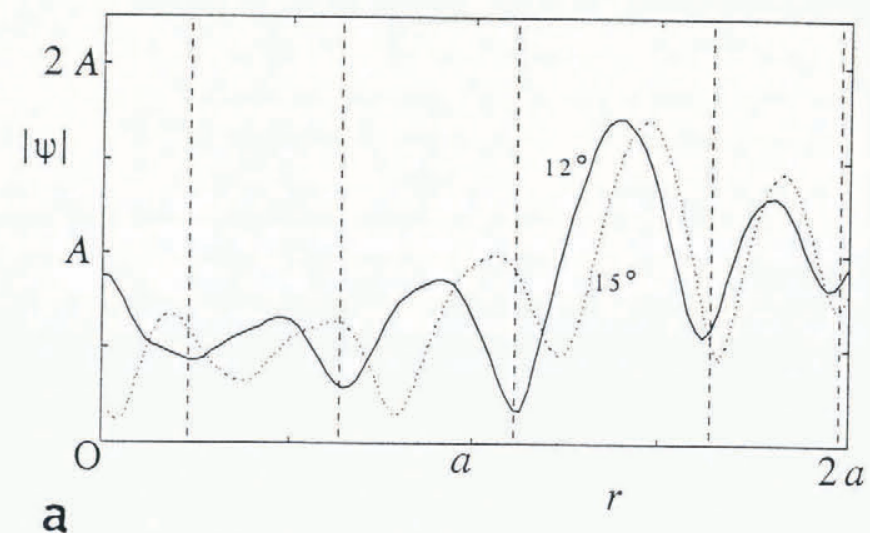

a

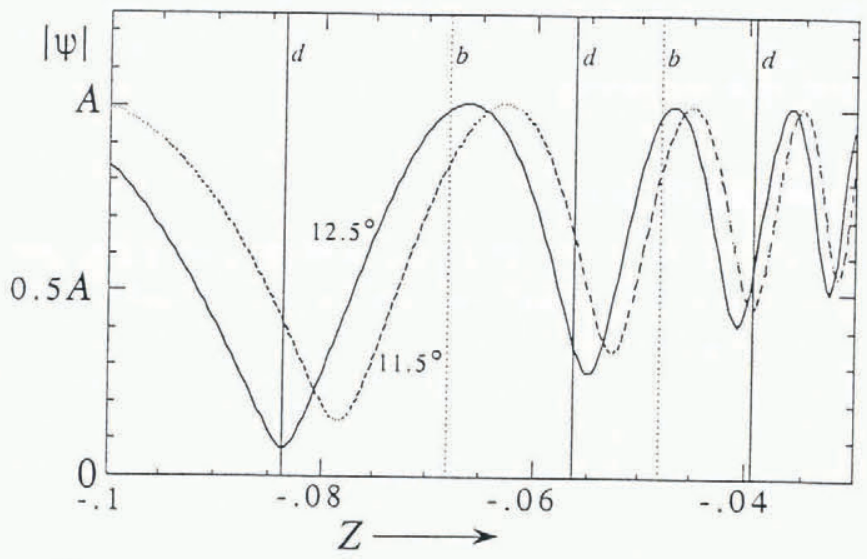

C
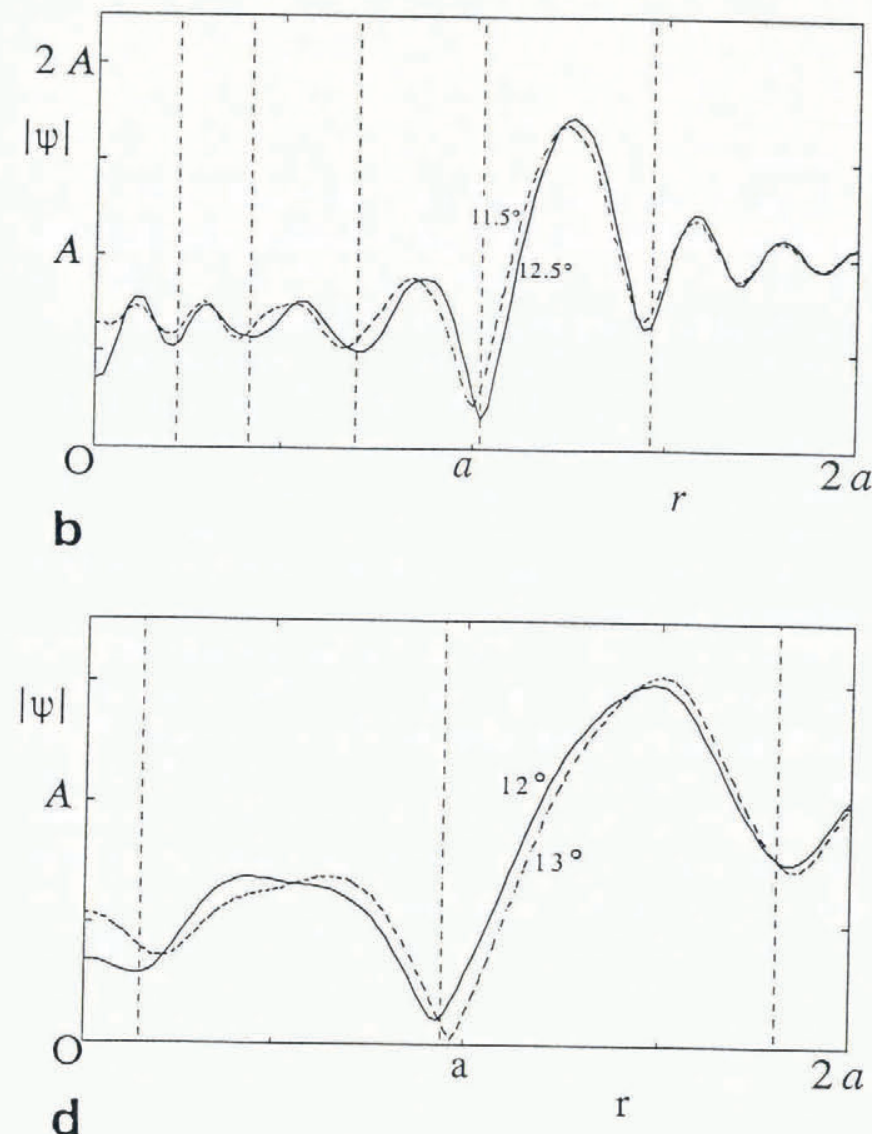

Fig. 5. (a) and (b). Theoretical variation of amplitude $|\psi|$ with radial distance $r$, compared with the measured radii of the dark rings (shown by the vertical broken lines). (a) is for lens A, $z=-22.58 \mathrm{~mm}$, corresponding to Figure $4 \mathrm{a} ;(\mathrm{b})$ is for lens $A, z=-12.99 \mathrm{~mm}$, corresponding to Figure 4b. Curves are labelled with the value of $\theta$. (c) Lens A; theoretical variation of amplitude along the axis in the virtual field, compared with the observed $Z$ values (vertical broken lines) where the ring system has a dark, $d$, or bright, $b$, centre. (d) Like (a) and (b) but for lens B; $z=-12.99 \mathrm{~mm}$, corresponding to Figure $4 \mathrm{~b}$.

To deduce the dihedral angle $2 \theta$, the method was as follows. A clear photograph of lens A was selected and values of $U$ and $Z$ were calculated. To compute the diffraction pattern requires a knowledge of $n$, but this depends on $f$ which in turn depends on $\theta$, which we do not yet know. Therefore, a trial and error method was used. A trial value was taken for $\theta ; n$ was deduced from the formula, derived from Equations (2), (4) and (5),

$$
n=\frac{2}{\lambda} a\left(\mu_{\mathrm{iw}}-1\right) \sin \theta
$$

and the section through the amplitude pattern corresponding to $Z$ was computed. This was compared with the observed pattern. $\theta$ was then varied until a satisfactory fit was achieved. Note that the value thus obtained is for a single lens and a single value of $Z$.

A check on the method was then made by choosing another value of $Z$ (i.e. another photograph, showing a quite different ring system) and repeating the exercise, thus obtaining another value of $\theta$, which should agree with the first. However, both values used the same value of $a$, the lens radius, and so are liable to a systematic error from this source. Therefore, we moved to the second lens $\mathrm{B}$ with four different values of $Z$, and thereby obtained four new values of $\theta$. The best value of $\theta$ was then deduced.

With lens A the measured values of distances in Equation (8) gave $u=1.58 \mathrm{~m}$. The wavelength used (sodium light) was $0.5893 \mu \mathrm{m}$ in free space, and therefore in ice $\lambda=0.4500 \mu \mathrm{m}$.(since $\mu_{\mathrm{i}}=1.3096$ ). Then, from Equation (4), $U=6.839$. A photograph (Fig. 4a) was chosen at $z=-22.58 \mathrm{~mm}$ (this is in the virtual field), which gives $Z=-0.0976$. We started with trial values $\theta=15^{\circ}$ and $18^{\circ}$, and found that $\theta \approx 12^{\circ}(n=3.111)$ would be needed to fit the observed ring sizes. Figure $5 \mathrm{a}$, which compares the computed amplitude as a function of $R$ with the observed positions of the dark rings, illustrates this; while both $\theta=12^{\circ}$ and $15^{\circ}$ fit the outer two rings, $\theta=12^{\circ}$ fits the inner ones as well. Moreover, for $\theta=12^{\circ}$, unlike $\theta=15^{\circ}$, the computed centre is bright and the darkest ring is the third one, as observed.

As a check, another photograph (Fig. 4b) was used, with the same lens but with $Z=-0.0562$. There are now more, and sharper, rings. Figure $5 \mathrm{~b}$ shows that $\theta=$ $12.5^{\circ}$ fits better than $11.5^{\circ}$, mainly because the former value reproduces the observed dark centre better. This conclusion is reinforced by Figure $5 c$, which shows the theoretical amplitude variation along the axis, compared with the observed distances where the ring system has a 
bright or a dark centre. $\theta=12.5^{\circ}$ is a good fit, while $\theta=$ $11.5^{\circ}$ is decidedly poorer. The centres of the ring systems are, in fact, the feature of the diffraction patterns most sensitive to $\theta$.

Lens B was now used with photographs at $Z=$ $-0.0799,-0.1072,-0.1618$ and -0.2007 , and trial values of $\theta=12^{\circ}(n=1.646)$ and $13^{\circ}(n=1.781)$. $u$ was the same as before, but the different value of $a$ now gives $U=24.43$. The clearest observations were for $Z=-0.2007$ (Fig. 4b). A central bright spot is just visible on the original photograph. Comparison with the curves in Figure $5 \mathrm{~d}$ for this $Z$ value suggests a value of $\theta$ between $12^{\circ}$ and $13^{\circ}$. We concluded from all the observations on this lens that $\theta$ for lens $\mathrm{B}$ is not measurably different from $\theta$ for lens $\mathrm{A}$.

The main sources of error are the measurements of the lens radii $a$ and the ring radii. Taking account of these errors, our final value for these two lenses, based on all the measurements, is $\theta=12.5 \pm 0.5^{\circ}$. To achieve significantly higher accuracy, one would have to consider the birefringence of the ice, since the maximum birefringence $\Delta \mu_{\mathrm{iw}}=0.0011$ corresponds to a difference in the deduced $\theta$ of $0.8^{\circ}$. Our value of $\theta$ is $26 \%$ lower than that found by Walford and others (1987), namely $16.8^{\circ}$. The difference is entirely accounted for by the effect of diffraction on the position of the apparent focus, as noted in section 1 .

\section{THE VARIABILITY OF DIHEDRAL ANGLE}

Formula (1) for $\theta$ contains the two surface energies $\gamma_{g b}$ and $\gamma_{\mathrm{iw}}$. The energy $\gamma_{\mathrm{gb}}$ of a boundary between two grains in any polycrystal is known to depend on the relative crystallographic orientation of the grains. For example, when there is a good fit between the lattices, the energy is abnormally low, giving rise to a downwardpointing cusp, that is, a singular minimum, in the curve of energy versus misorientation angle (e.g. Pumphrey, 1976). The present observations, like the earlier ones, detected no marked systematic (i.e. crystallographic) ellipticity or astigmatism in the lenses, although some of them (including lens A) were measurably non-circular; therefore, each lens face was apparently spherical on average, in spite of the variation of crystallographic orientation over it. This means that $\gamma_{\text {iw }}$ can be only weakly dependent on the orientation of the interface. One would therefore expect, as a good approximation, that $\theta$ would be constant for any one grain boundary. Both the lenses measured in the present work were in the same grain boundary, and this is no doubt why the values of $\theta$ agree so well. However, observations by Morris (1972) and observations on the veins at the junctions of three grains (Mader, in press) show that, while $\theta$ is usually approximately constant, there are occasional boundaries where $\theta$ can take quite different values, as high as $52^{\circ}$. These must be the low-energy boundaries corresponding either to low angles of misorientation, or to a good fit of the lattices.

In summary, the method of finding dihedral angle described here is very accurate for measuring $\theta$ in a given grain boundary. We believe the grain boundary chosen for study was not unrepresentative, but it has to be remembered that some grain boundaries would have given very different values.

\section{REFERENCES}

Farnell, G. W. 1957. Calculated intensity and phase distribution in the image space of a microwave lens. Can. J. Phys., 35, 777-783.

Mader, H. In press. Observations of the water-vein system in polycrystalline ice. J. Glaciol.

Morris, E.M. 1972. The sliding of ice over rock and other substances. (Ph.D. thesis, University of Bristol.)

Nye, J.F. 1991. Diffraction by a small unstopped lens. J. Modern Optics, 38(4), 743-754.

Nye, J.F. and F. C. Frank. 1973. Hydrology of the intergranular veins in a temperate glacier. International Association of Scientific Hydrology Publication 95 (Symposium at Cambridge $1969-$ Hydrology of Glaciers), 157-161.

Nye, J.F. and S. Mae. 1972. The effect of non-hydrostatic stress on intergranular water veins and lenses in ice. J. Glaciol., 11(61), 81-101.

Pumphrey, P. H. 1976. Special high angle grain boundaries. In Chadwick, G. A. and D. A. Smith, eds. Grain boundary structure and properties. London, Academic Press, 139-200.

Walford, M. E. R., D. W. Roberts and I. Hill. 1987. Optical measurements of water lenses in ice. J. Glaciol., 33(114), 159-161.

The accuracy of references in the text and in this list is the responsibility of the authors, to whom queries should be addressed. 